Bull. Austral. Math. Soc.

Vol. 40 (1989) [331-332]

\title{
ON SOME GROUPS WITH TRIVIAL MULTIPLICATOR
}

JAMES WIEGOLD

\section{For Bernhard Neumann, with respect and affection}

\author{
Scene: $\quad$ B.H. Neumann's office, in the University of Manchester; \\ an M.Sc. tutorial. \\ Date: late 1954. \\ Dramatis Persunae: B.Il. Neumann and James Wiegold. \\ B.H.N.: "So you see, the Schur multiplicator is an important tool \\ for the BFC-problem and others; thou shouldst definitely \\ go forth and multiplicate." \\ J.W.: \\ "I Schur should."
}

I discussed the sort of thing Bernhard had in mind concerning the BFC-problem in [3], an article written for his sixieth birthday. This vigesennial contribution deals with one of the "and others", namely the zero deficiency problem. In his article [1] bearing the same title as this one, Bernhard gave 2-generator 2-relator presentations of some metacyclic groups; if one distils and formalises the process used there, one gets the following result, to be used later in constructing some zero-deficiency finile $p$-groups.

THEOREM. Let $m$ and $n$ be positive integers and $w$ a two-variable word. The group $G=\left\langle a, b \mid a^{m}=b^{n}=w(a, b)\right\rangle$ is finite if and only if $G / G^{\prime}$ and $H=$ $\left\langle a, b \mid a^{m}=b^{n}=w(a, b)=1\right\rangle$ are finite. If $G / G^{\prime}$ and $H$ are finite $\pi$-groups for sorne set $\pi$ of primes, so is $G$.

Proof: One way around everything is obvious. Suppose that $G / G^{\prime}$ and $H$ are fituite $\pi$-groups. Since $a^{m}$ is central in $G$ and $G /\left\langle a^{m}\right\rangle \cong H$, it follows that $G^{\prime} \cap\left\langle a^{m}\right\rangle$ is a finite $\pi$-group, being a homomorphic inage of the inultiplicator of $H$. But $a^{s} \in G^{\prime}$ for some $\pi$-number $s$, and $a^{m s}$ has $\pi$-order since $a^{m s} \in G^{\prime} \cap\left\langle a^{m}\right\rangle$. Thus $\left\langle a^{m}\right\rangle$ is a finite $\pi$-group, and therefore so is $G$.

Of course, the theorem has a version for groups on more than two generators and/or more than one relator. However, the main interest is in zero deficiency groups.

Received 6 April, 1989

Copyright Clearance Centre, Inc. Serial-fee code: 0004-9729/89 $\$$ A2.00+0.00. 
Robertson [2] has constructed some finite non-metacyclic 2-groups of zero deficiency and high nilpotency class; as far as I know, 2 is the only prime where this has been achieved to date. The theorem allows us to make some suitable 3-groups.

Example 1. The group $G=\left\langle a, b \mid a^{3^{n}}=b^{3}=\left[a, a^{b}\right]\right\rangle$ is a finite 9-group of nilpotency class $2 n+2$.

Proof: Clearly, $G / G^{\prime}$ is a finite 3-group. But the corresponding $H=$ $\left\langle a, b \mid a^{3^{n}}=b^{3}=\left[a, a^{b}\right]=1\right\rangle$ is nothing but $Z_{3^{n}} \mid Z_{3}$ thinly disguised; the relation $\left[a, a^{b}\right]=1$ yields $1=\left[a, a^{b}\right]^{b}=\left[a^{b}, a^{b^{2}}\right]$ and $1=\left[a^{b}, a^{b^{2}}\right]^{b}=\left[a^{b^{2}}, a\right]$, so that $\left\langle a, a^{b}, a^{b^{2}}\right\rangle$ is a commutative subgroup of $H$. The class of $H$ is $2 n+1$, and the claim about the class of $G$ follows after a tedious calculation.

Of course, the group oblained by replacing 3 by 2 in Example 1 is a finite 2-group of high class. Here is a slightly more complicated example.

Example 2. The group $G=\left\langle a, b \mid a^{2^{n}}=b^{2}=\left[a^{b}, a, a\right]\right\rangle$ is a finite 2-group of class $n+2$.

ProOF: Here $H=\left\langle a, b \mid a^{2^{n}}=b^{2}=\left[a^{b}, a, a\right]=1\right\rangle$ is the second nilpotent wreath product of $Z_{2^{n}}$ by $Z_{2}$; for $1=\left[a^{b}, a, a\right] \Rightarrow 1=\left[a^{b}, a, a\right]^{b}=\left[a, a^{b}, a^{b}\right]$, so that the normal closure $\left\langle a, a^{b}\right\rangle$ of $a$ is nilpotent of class two. I shall once more omit the class-claim.

Like all other methods attempted so far, that presented here appears to be hopeless for constructing zero-deficiency groups of high solubility length. Further, I think there is enough lack of evidence around to make a final point:

Conjecture. For each $p \geq 5$, zero-deficiency finite $p$-groups have $p$-bounded nilpotency classes.

I shall try to confirm or deny this in readiness for October 2009.

\section{References}

[1] B.H. Neumann, 'On some groups with trivial multiplicator', Publ. Math. Debrecen 4 (1956), 190-194.

[2] Edmund F. Robertson, 'A comment on finite nilpotent groups of deficiency zero', Canad. Math. Bull. 23 (3) (1980), 313-316.

[3] James Wiegold, 'Commulator subgroups of finite p-groups', J. Austral. Math. Soc. 1 (1969), 480-484.

School of Mathematics

University of Wales

College of Cindift

Senghenydd Road

Cardift CF2 4AG

United Kingdom 